\title{
Intense acoustic bursts as a signal-enhancement mechanism in ultrasound-modulated optical tomography
}

\author{
Chulhong Kim, Roger J. Zemp, and Lihong V. Wang \\ Optical Imaging Laboratory, Department of Biomedical Engineering, 3120 TAMU, Texas A\&M University, \\ College Station, Texas 77843-3120
}

Received March 21, 2006; accepted May 8, 2006; posted May 25, 2006 (Doc. ID 69198); published July 25, 2006

\begin{abstract}
Biophotonic imaging with ultrasound-modulated optical tomography (UOT) promises ultrasonically resolved imaging in biological tissues. A key challenge in this imaging technique is a low signal-to-noise ratio (SNR). We show significant UOT signal enhancement by using intense time-gated acoustic bursts. A CCD camera captured the speckle pattern from a laser-illuminated tissue phantom. Differences in speckle contrast were observed when ultrasonic bursts were applied, compared with when no ultrasound was applied. When CCD triggering was synchronized with burst initiation, acoustic-radiation-force-induced displacements were detected. To avoid mechanical contrast in UOT images, the CCD camera acquisition was delayed several milliseconds until transient effects of acoustic radiation force attenuated to a satisfactory level. The SNR of our system was sufficiently high to provide an image pixel per acoustic burst without signal averaging. Because of the substantially improved SNR, the use of intense acoustic bursts is a promising signal enhancement strategy for UOT. (C) 2006 Optical Society of America

OCIS codes: $030.1670,110.6150,110.7050,110.7170,170.3880$.
\end{abstract}

Ultrasound-modulated optical tomography ${ }^{1,2}$ (UOT) is a novel imaging technique that offers strong optical contrast with high ultrasonic spatial resolution. The principle of this technique is that a highcoherence laser source illuminates a light-scattering medium while the medium is insonified with ultrasound. Light passing through the ultrasonic focal volume is tagged by both ultrasound-induced particle displacement and changes in refractive index such that each detected optical speckle spot has a timevarying modulation component due to the ultrasonic interaction. ${ }^{3-5}$ By measuring the degree of modulation at each ultrasonic focal volume location, images representative of the optical properties of the tissue may be formed. A major difficulty in UOT is the low signal-to-noise ratio (SNR) due to low modulation efficiency and uncorrelated phases between laser speckle grains. A number of groups have developed detection methods, including parallel detection with CCD cameras, ${ }^{6-8}$ Fabry-Perot-interferometer-based detection, ${ }^{9}$ photorefractive-crystal-based detection, ${ }^{10}$ and speckle-contrast detection, ${ }^{11}$ to overcome this problem.

In this Letter, using a speckle-contrast detection technique ${ }^{11}$ we explore the use of intense ultrasound bursts instead of the use of continuous-wave (CW) or pulsed ultrasound. ${ }^{12}$ By reducing the duty cycle of the ultrasonic transducer, it is possible to use much higher pressure. This acoustic burst application acts to displace optical scatterers by as much as several micrometers, ${ }^{13}$ compared with angstrom- or nanometer-scale particle displacements that are due to $\mathrm{CW}$ ultrasound with lower pressure. Such displacements serve to enhance modulation in the lightspeckle pattern from the sample. We therefore predicted that intense acoustic bursts would induce greater particle displacements and thus provide a larger source of modulation. We show for the first time to our knowledge that the application of intense acoustic bursts is a significant mechanism of signalenhancement and that this effect can provide spatial resolution comparable with previous $\mathrm{CW}$ techniques.

During time scales of the order of a millisecond, intense acoustic bursts induce localized tissue displacements due to acoustic radiation force generated by an inherent momentum transfer when ultrasound interacts with an interface of mismatched acoustic impedance, with ultrasonic scatterers, or with ultrasonic absorbers. ${ }^{13,14}$ We experimentally demonstrate slight, but statistically significant, radiation-force enhancement of UOT signals beyond pure ultrasound mechanisms. This transient acoustic radiation force (TARF), however, may provide a mechanical contrast in UOT images. To minimize this effect, we trigger our CCD camera image acquisition several milliseconds after the acoustic burst generation, such that transient tissue motion due to acoustic radiation force has been sufficiently damped, and pure ultrasound mechanisms dominate.

We compare signal levels with three mechanisms: the CW ultrasound mechanism with low pressure, the TARF mechanism at the initiation of an ultrasonic burst, and the steady-state effect at nearly the end of an ultrasonic burst. To demonstrate the advantage of a higher SNR, we show high-contrast UOT images of phantoms without signal averaging, an important step for moving UOT closer to in vivo imaging studies.

The experimental setup is shown in Fig. 1. The laser light (Coherent, Verdi; $532 \mathrm{~nm}$ wavelength) was projected on a tissue-mimicking phantom. The average laser power delivered to the sample during the propagation of an ultrasonic burst was $\sim 12 \mathrm{~mW} / \mathrm{cm}^{2}$, a value within safety limits. Acoustic waves were pro- 


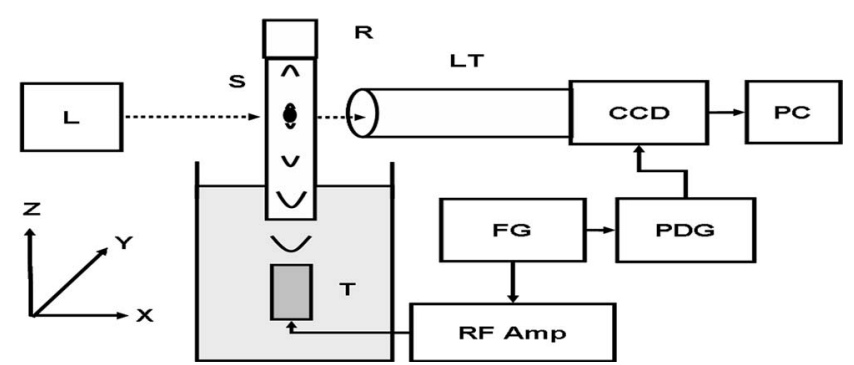

Fig. 1. Experimental setup: L, laser; CCD, CCD camera; $\mathrm{RF}$ amp, RF amplifier; FG, function generator; PDG, pulsedelay generator; T, ultrasound transducer; S, sample; LT, lens tube; R, rubber; PC, personal computer.

duced by a focused ultrasound transducer (Ultran, VHP100-1-138; $1 \mathrm{MHz}$ central frequency, $25.4 \mathrm{~mm}$ lens diameter, $38 \mathrm{~mm}$ focal length). The focal zone of the ultrasonic waves was $\sim 2 \mathrm{~mm}$ wide and $\sim 20 \mathrm{~mm}$ long. The ultrasound peak pressure at the focal spot for the image experiments was applied up to 1.5 $\mathrm{MPa}$, so the mechanical index at this frequency was $\sim 1.5$, which is within typical safety limits for diagnostic ultrasound. ${ }^{15}$ We used a gelatin-cornstarch phantom (10\% gelatin and 10\% cornstarch by weight; the cornstarch acted as both the ultrasonic absorbers and the optical scatterers) whose reduced scattering coefficient was $\sim 9.2 \mathrm{~cm}^{-1}$ measured by an obliqueincidence reflectometer. ${ }^{16}$ The light transmitted through the sample yielded a speckle pattern that was detected by a digital CCD camera (Basler, A312f; 12 -bit, $640 \times 480$ pixels). A lens tube was mounted on the CCD camera to match the average speckle size to the size of a CCD pixel and block all other disturbances in the transmitted light. ${ }^{11} \mathrm{~A}$ function generator (Agilent, 33250A) synthesized $8 \mathrm{~ms}$ bursts that were subsequently amplified by an rf amplifier (ENI, Inc., 325LA) to drive the ultrasound transducer. Burst initiation triggered a pulse-delay generator (Stanford Research, DG535) that produced two CCD trigger pulses for each burst. One burst-synchronized frame was captured on the CCD camera, after which an image was taken with no ultrasound burst. Every second we measured the laser speckle contrast on the CCD surface with and without the ultrasound modulation. CCD image capture can be accomplished either at the initiation of an $8 \mathrm{~ms}$ burst (to see the TARF effect) or near the end of the burst (a CCD trigger delay of $6 \mathrm{~ms}$ ), where the TARF effect has attenuated (to see the steady-state effect, and we refer to this as quasi $\mathrm{CW}$ ). The exposure time of the CCD camera was set to $2 \mathrm{~ms}$. We defined our signal as the change in speckle contrast between ultrasound on and off in speckle-contrast measurements. This measure reflects the amount of modulated light originating from the ultrasonic focal volume.

To examine signal enhancement by intense ultrasonic bursts, we measured the signal with increasing ultrasonic pressure within the current safety limit [Fig. 2(a)]. As the pressure mounts, the signal increases, and the difference between TARF and quasi-CW regimes become larger. We compared the SNRs of three methods: $0.3 \mathrm{MPa} \mathrm{CW}, 1.5 \mathrm{MPa}$ quasi $\mathrm{CW}$, and $1.5 \mathrm{MPa}$ TARF. The SNR, defined as the mean of the change in speckle contrast between ultrasound on and off divided by its root-mean-square standard deviations, for $1.5 \mathrm{MPa}$ quasi $\mathrm{CW}$ was $260 \pm 4$ (standard error) compared with $287 \pm 5$ for 1.5 $\mathrm{MPa}$ TARF and $32 \pm 1$ with $0.3 \mathrm{MPa} \mathrm{CW}$ [Fig. 2(a)]. The SNR enhancement of the quasi-CW mechanism over the CW mechanism is about 8 times, compared with the SNR enhancement of $10 \%$ with the TARF mechanism over the quasi-CW mechanism. The quasi-CW method can provide a SNR comparable with that of the TARF method without offering any mechanical contrast. In addition, comparison of signals with different CCD trigger delay times can distinguish the effects of the TARF and the quasi-CW methods [Fig. 2(b)]. To show this, we applied a $14 \mathrm{~ms}$ ultrasonic burst of $\sim 2 \mathrm{MPa}$ peak pressure and triggered the CCD camera at $0,2,4, \ldots, 12 \mathrm{~ms}$ delays following the burst initiation. The largest signal is generated when the burst and the CCD trigger are synchronized. After this point, the TARF effect attenuates and the signal decreases as it approaches a steady-state condition, where the quasi-CW mechanism is more dominant than the TARF mechanism [Fig. 2(b)]. Although the TARF mechanism offers signal enhancement over the quasi-CW mechanism at the same pressure, the relative enhancement is only $11 \%$.

To investigate the potential of using intense bursts for optical imaging, we scanned phantoms and compared image contrast and lateral spatial resolution by using $0.3 \mathrm{MPa} \mathrm{CW}, 1.5 \mathrm{MPa} \mathrm{TARF}$, and $1.5 \mathrm{MPa}$ quasi-CW mechanisms. We imaged a gelatincornstarch phantom with two objects dyed with Trypan Blue $(2.1 \mathrm{~mm} \times 2.6 \mathrm{~mm} \times 17 \mathrm{~mm}$ and $2.3 \mathrm{~mm}$ $\times 2.5 \mathrm{~mm} \times 17 \mathrm{~mm}$ along the $X, Y$, and $Z$ axes, respectively) separated by $12 \mathrm{~mm}$ from center to center. The mechanical properties of the two objects were identical to the surrounding phantom. As the ultrasonic pressure increased, the signal likewise increased. In addition, two absorbing objects are clearly

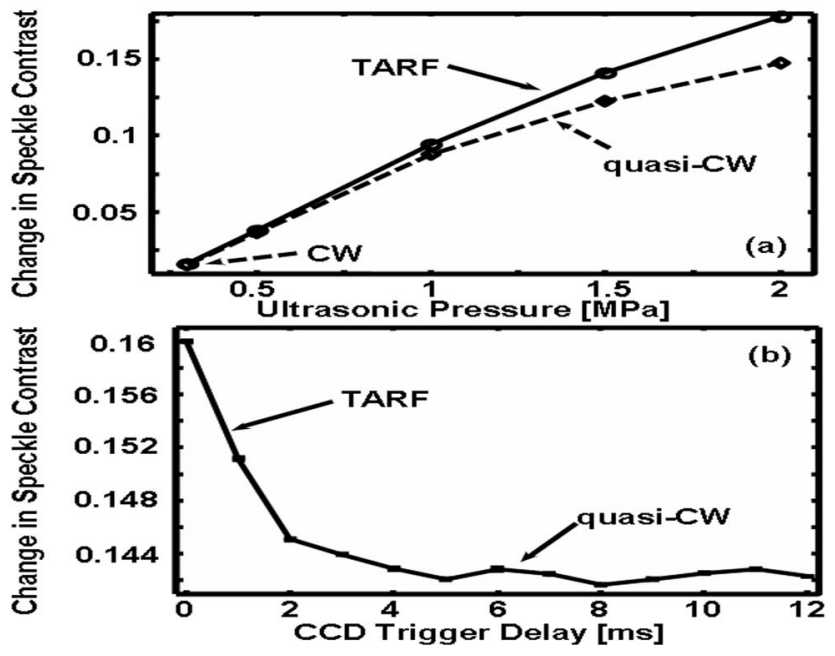

Fig. 2. Comparison of signals defined as changes in speckle contrast (a) for TARF and quasi-CW mechanisms applied at different ultrasonic pressures and (b) in TARF and quasi-CW regimes, varying CCD trigger delay times with an ultrasonic pressure of $2 \mathrm{MPa}$. 


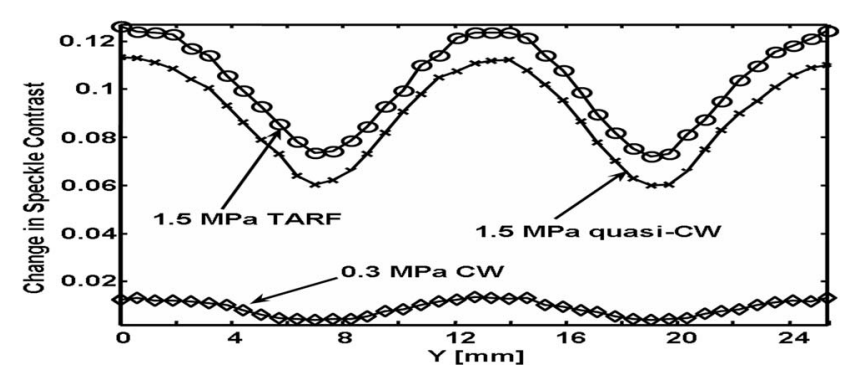

Fig. 3. Comparison of 1D images of two objects dyed with Trypan Blue in a $1.9 \mathrm{~cm}$ thick phantom with varying ultrasonic pressures.
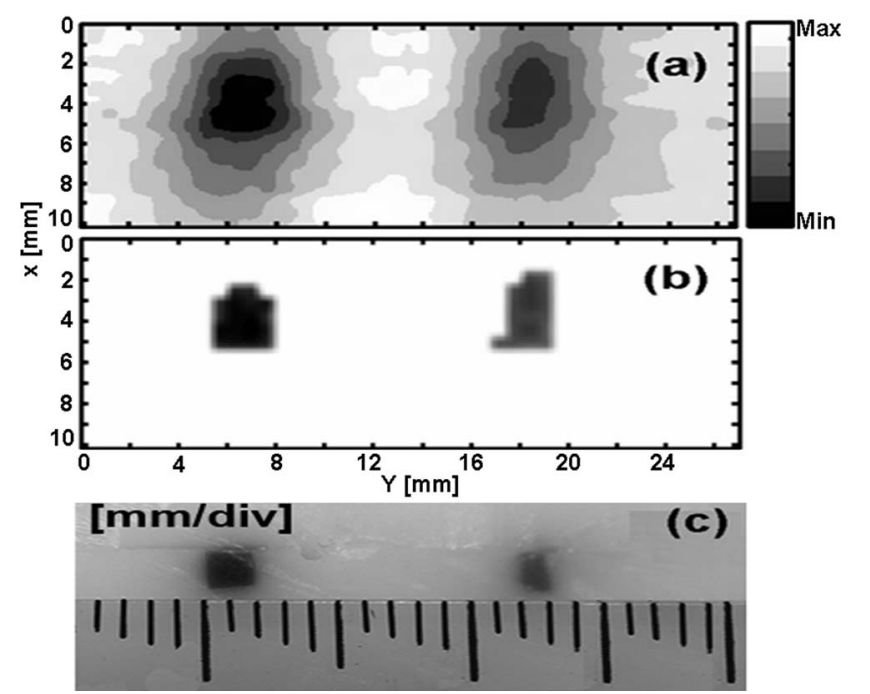

Fig. 4. (a) 2D image of two objects dyed with Trypan Blue embedded in a $1.9 \mathrm{~cm}$ thick phantom acquired by using the 1.5 MPa quasi-CW mechanism without any signal averaging. (b) 2D image with $15 \%$ thresholding. (c) Photograph of the phantom.

seen in Fig. 3. At the same pressure, the signal with the TARF mechanism is only $12 \%$ larger than the signal with the quasi-CW mechanism. However, signals from both the TARF and the quasi-CW methods are 10 times larger than the signal with the CW method. Even though the relative image contrast (the difference between the minimum and maximum values divided by the maximum value) of the $1.5 \mathrm{MPa}$ images is lower than the $0.3 \mathrm{MPa} \mathrm{CW}$ images, the absolute image contrast (subtracting the minimum value from the maximum value) is five times greater. Lateral spatial resolution, defined as the one-way distance between $25 \%$ and $75 \%$ of the maximum over the minimum, is approximately $2 \mathrm{~mm}$ with all three mechanisms, which is comparable with the ultrasonic beam waist.

Figure 4(a) shows a 2D UOT image obtained from a $19 \mathrm{~mm}$ thick phantom [Fig. 4(c)] containing two objects dyed with Trypan Blue separated by $11 \mathrm{~mm}$. The sizes of the two objects were $2.0 \mathrm{~mm} \times 2.0 \mathrm{~mm}$ $\times 12.3 \mathrm{~mm}$ and $2.0 \mathrm{~mm} \times 1.3 \mathrm{~mm} \times 12.3 \mathrm{~mm}$ [Fig. 4(c)]. We used the quasi-CW mechanism with an ultrasonic pressure of $1.5 \mathrm{MPa}$ and a CCD trigger delay of $6 \mathrm{~ms}$. To generate a 2D image, only 1 pair of on-off measurements were taken for each image pixel. In the image, the objects are clearly seen with a relative image contrast of $31 \%$ and a lateral spatial resolution of $\sim 2.0 \mathrm{~mm}$ [Fig. 4(a)]. Clear delineation of the embedded objects is seen with 15\% thresholding [Fig. 4(b)]. In addition, the second object is narrower than the first object along the $Y$ direction [Fig. 4(b)], which exactly matches the photograph [Fig. 4(c)].

In summary, this research has demonstrated that intense acoustic bursts can provide significant UOT signal enhancement, higher absolute image contrast, and comparable spatial resolution compared with lower-pressure CW ultrasound. Because of the benefits of the higher SNR, the scanning time may be reduced by 1-2 orders of magnitude, and UOT scanning can be performed without signal averaging. Furthermore, the imaging system was extremely robust to environmental disturbances. This technique may be integrated with conventional UOT detection techniques such as parallel detection with CCD cameras, Fabry-Perot-interferometer-based detection, and photorefractive-crystal-based detection.

We thank X. Xiao, S. Sakadžić, and A. Garcia-Uribe for fruitful scientific discussions. This research was supported by National Institutes of Health grant CA094267. L. Wang's e-mail address is lwang @tamu.edu.

\section{References}

1. F. A. Marks, H. W. Tomlinson, and G. W. Brooksby, in Proc. SPIE 1888, 500 (1993).

2. L.-H. V. Wang, S. L. Jacques, and X. Zhao, Opt. Lett. 20, 629 (1995).

3. W. Leutz and G. Maret, Physica B 204, 14 (1995).

4. L.-H. V. Wang, Phys. Rev. Lett. 87, 043903 (2001).

5. S. Sakadžic and L.-H. V. Wang, Phys. Rev. E 66, 026603 (2002).

6. S. Leveque, A. C. Boccara, M. Lebec, and H. SaintJalmes, Opt. Lett. 24, 181 (1999).

7. G. Yao, S.-L. Jiao, and L.-H. V. Wang, Opt. Lett. 25, 734 (2000).

8. M. Gross, P. Goy, and M. Al-Koussa, Opt. Lett. 28, 2482 (2003).

9. S. Sakadžić and L.-H. V. Wang, Opt. Lett. 29, 2770 (2004).

10. T. W. Murray, L. Sui, G. Maguluri, R. A. Roy, A. Nieva, F. Blonigen, and C. A. DiMarzio, Opt. Lett. 29, 2509 (2004).

11. J. Li, G. Ku, and L.-H. V. Wang, Appl. Opt. 41, 6030 (2002).

12. A. Lev and B. G. Sfez, Opt. Lett. 28, 1549 (2003).

13. K. Nightingale, M. S. Soo, R. Nightingale, and G. Trahey, Ultrasound Med. Biol. 28, 227 (2002).

14. G. R. Torr, Am. J. Phys. 52, 402 (1984).

15. D. Dalecki, Annu. Rev. Biomed. Eng. 6, 18.1 (2004).

16. G. Marquez and L.-H. V. Wang, Opt. Express 1, 454 (1997). 